\title{
Una mirada a la concepción del sujeto en la educación: más allá de lo racional
}

Juan José Burgos Acosta*

Resumen. La comprensión del sujeto desde una perspectiva anclada en dimensiones como la racionalidad, la afectividad y el valor del pensamiento subjetivo conduce hacia un marco de interpretación que transita por caminos distintos a los propuestos por la racionalidad instrumental, pues al centrarse en la individualidad del sujeto y su capacidad para construir mundos posibles a partir de la interpretación de su propio presente, la acción individual y colectiva cobra nuevos sentidos para realizar acciones encaminadas a la superación de las tradicionales formas míticas de vivir.

Palabras Claves. Sujeto, Racionalidad, Afectividad. Acción Colectiva.

Doctor Honoris Causa en Filosofía de la Educación, otorgado por el Consejo Iberoamericano en Honor a la Calidad Educativa. Doctorado en Educación Estudiante de primer año Universidad Santo Tomás. Magíster en Educación, Magíster en Estudios Políticos, Estudios Superiores en Teología y Licenciado en Teología de la Pontificia Universidad Javeriana, Licenciado en Filosofía de la Universidad Santo Tomás. Chasjjba14@yahoo.es 
Abstract. The understanding of the subject from a perspective rooted in dimensions such as rationality, the emotions and the value of subjective thought leads to a framework of interpretation that goes through different paths to those proposed by instrumental rationality, as the focus on the individuality of the subject and their ability to construct possible worlds from the interpretation of its own present, individual and collective action takes on new meanings for actions aimed at overcoming traditional mythical ways of living.

Keywords. Subject, Rationality, Emotional. Collective Action.

Nosotros hablamos como si lo racional tuviese un fundamento trascendental que le da validez universal independiente de lo que nosotros hacemos como seres vivos. Esto no es así. Todo sistema racional se funda en premisas fundamentales aceptadas a priori, aceptadas porque sí, aceptadas porque a uno le gustan, aceptadas porque uno las acepta simplemente desde sus preferencias.

Humberto Maturana

\section{Introducción}

Esta primera década del siglo XXI que acaba de perecer se ha caracterizado especialmente por la acentuación de la llamada globalización con unas manifestaciones especiales que bien valen la pena tener en cuenta en la construcción del concepto del sujeto. La privatización de la violencia a través del terrorismo, el hecho de que cada vez más los conflictos sean internos y no externos, la naturaleza visible de la amenaza terrorista y la multiplicación de amenazas no políticas como la pandemia universal y, especialmente el cambio climático, sentido con furor en todos los países del mundo, han contribuido a un sentimiento de inseguridad, vulnerabilidad y miedo en las poblaciones, que obligan con urgencia a 
repensar y a resignificar distintas formas en que los seres humanos, no sólo piensan, sino actúan para resolver tales problemas. Por eso lo que se pretende desarrollar en este texto es la necesidad de desplazar la concepción más poderosa que ha reinado sobre el sujeto particular desde la Modernidad: la visión racional, que lo ha convertido en un instrumento mercantil, y abrir otras posibilidades, como lo emocional-afectivo, que constituyen materia prima en la construcción de sujetos más solidarios y preocupados por los principales problemas que aquejan a la humanidad, y cómo desde la educación es posible la emergencia de sociedades más incluyentes y armónicas.

\section{Una trilogía que enceguece}

Hay una trilogía que ha logrado "enceguecer" de forma invisible y que es necesario superar en el abordaje de la concepción del sujeto en la educación. La trilogía del mito de la cultura patriarcal, del mito de la objetividad y del mito de la racionalidad, que se ha incorporado profundamente en los individuos y conduce como una mano invisible la forma de ver el mundo y de actuar en él, generando unas cegueras y unas predisposiciones que se constituyen en los principales obstáculos en los procesos de reconfiguración de las relaciones sociales y del establecimiento de sociedades más armónica que perduren en el tiempo.

Las problemáticas que ha venido generando el mito de la cultura patriarcal se expresan en el mundo occidental en donde se encuentran múltiples culturas locales, regionales y nacionales que tienen formas de expresiones particulares, originadas en el entrelazamiento dinámico de las especificidades propias del contexto sociogeográfico, los usos y prácticas ancestrales y la historia de interacciones transcurridas en esos ámbitos. Pero como un común denominador de todas estas culturas se encuentra un modo de vida que se caracteriza por estar centrado en la apropiación, el dominio de unos y el sometimiento de otros, la competencia, el 
control, la desconfianza y la guerra, que constituye un gran trasfondo de sentido que anima la existencia de las personas que viven esas culturas. Es una especie de cultura fundante, una cultura de culturas, una metacultura a la que algunos autores han denominado "cultura patriarcal", (Maturana, 2003, p. 36). Otros, como el "sistema capitalista" (Peresson, 2005, p. 22); que últimamente se expresa como neoliberalismo (Balbín y Arango, 2003, p. 102), se constituye de alguna manera en uno de los componentes de la llamada globalización.

El segundo mito que ha hecho daño a la concepción de sujeto en la educación, es el de la objetividad, el cual llena de certidumbres y verdades a la cultura, llevando a considerar que quienes opinan o ven el mundo de manera diferente a la suya están equivocados y por tanto deben ser ilustrados o presionados para que se pongan del lado "correcto", o que son perversos y actúan de mala fe, por lo cual se deben suprimir o acallar mediante métodos coercitivos. El mito de la objetividad ha estado en la base de muchos conflictos de orden religioso, étnico, político y de la cotidianidad, y es una de las talanqueras más recalcitrantes en la búsqueda de la construcción de ciudadanía, por cuanto ciega ante los criterios de validez que portan los otros, cierra a las múltiples miradas sobre el mundo, y al hacer creer que hay unos depositarios de la "verdad", los lleva a un sordo enfrentamiento entre certidumbres. Y es que la cuestión del estatuto ontológico de la realidad, es decir, las respuestas a las preguntas: iel sujeto qué puede conocer acerca de la realidad?, o en otros términos iqué tan real es la realidad que percibimos como sujetos?, si el mundo que se observa y con el cual interactúa, existe tal se percibe, es uno de los problemas más acuciantes para la humanidad, porque de la respuesta que se presente, depende el tipo de relaciones que se construyan con el entorno social y educativo, particularmente la aceptación o negación de las miradas o explicaciones de los otros sujetos; esto es, en el mantenimiento de una postura hegemónica o pluralista en las distintas construcciones que se ha hecho sobre el sujeto. 
La postura objetivista habla de un único mundo y en consecuencia de una única verdad, la cual consiste en el enunciado que explica y describe el mundo tal cual es. La función de la ciencia y de la actividad cognitiva se ubica en el develamiento de lo que está oculto por las apariencias externas de los fenómenos y de las cosas, en el descubrimiento de las leyes y causalidades que rigen y generan el mundo y sus procesos de cambio. El conocimiento por tanto es objetivo de suyo; de por sí, de manera que quienes operan en esta perspectiva aceptan que existe un solo dominio de realidad, es decir, un universo. Ante las diversas visiones de realidad que portan los demás sujetos (observadores) se produce una invalidez de su conocimiento, porque se considera que "no es objetivo". Ante esta forma de ver el mundo Maturana (2002, p. 22), expresa que "en ese camino argumentativo las explicaciones suponen la posesión de un acceso privilegiado a una realidad objetiva por el observador que explica, y por tanto la pretensión de conocimiento es una demanda de obediencia".

El mito de la racionalidad, por su parte, conduce a un reduccionismo que desconoce otras dimensiones fundamentales en la comprensión del sujeto, limita el panorama de las actuaciones socioeducativas a la esfera cognitiva, focalizándolas solamente hacia el suministro de información, a la ilustración y a la enseñanza de conceptos y teorías, con la consecuencia de que los aprendizajes quedan constreñidos únicamente a la aprehensión de nuevos discursos que ocultan y enmascaran con nuevas terminologías las viejas prácticas que se pretenden cambiar. Al afirmarse en la creencia de que los seres humanos orientan sus conductas exclusivamente por las valoraciones que determinan la mejor opción racional, por las argumentaciones lógicamente más sólidas y fundamentadas y/o por la referencia a principios axiológicos universalistas, o valores que indican cuáles son las acciones "correctas" o más convenientes que se deben seguir, los enfoques racionalistas quedan ciegos ante los otros dos subsistemas (el corporal y el emocional) que junto al lenguaje (la razón) componen al sujeto. 
Cuando se pegunta qué es lo específico del sujeto humano, la respuesta común es que, es animal racional. Y en efecto lo es. Sólo que se ha magnificado este rasgo, lo cual hace que se pase por alto y se desvalorice el hecho de que como seres biológicos, los sujetos también son seres emocionales y que, en ese sentido las acciones necesariamente se entrelazan entre razón y emoción. Se puede afirmar que cuando el sujeto está en el dominio de una emoción se pueden hacer unas cosas y otras no, y que al mismo tiempo se rechaza o se acepta un argumento bajo otra emoción, por ejemplo, "me gusta su planteamiento y por eso lo acepto", "no me gusta sus argumentos porque...", etc.; manifestándose así la dinámica entre emoción y razón. A propósito del asunto, Echeverría (1996) considera que:

Desde el comienzo, sin embargo, hubo reacciones contrarias a esta concepción del ser humano, especialmente en cuanto al papel asignado a nuestras emociones, los así llamados "asuntos del corazón". Blaise Pascal, filósofo francés contemporáneo de Descartes, nos advirtió que el "corazón tiene sus razones que la razón desconoce". Pero a pesar de la advertencia, el cartesianismo se convirtió en nuestra forma predominante de comprender el fenómeno humano. Sostenemos que para comprender la acción humana, debemos prestar cuidadosa atención a nuestra vida emocional. Postulamos que nuestras emociones son determinantes básicas de lo que podemos o no lograr en los dominios del aprendizaje y/o sociabilidad" (pp. 255-256).

Cuando las personas no logran discernir y hacerse cargo de que son portadoras del emocionar propio de la cultura en la que viven y que ésta modula momento a momento, espontánea e imperceptiblemente sus deseos, gustos, aspiraciones y evaluaciones de lo que les resulta gratificante o aversivo, sus prácticas resultan incongruentes con sus discursos de convivencia y educación democrática, de modo que la construcción cotidiana que hacen en la ejecución de los proyectos educativos resulta contraria a 
los propósitos formulados, y con frecuencia terminan reforzando lo que en la letra desean cambiar. Morín (2000, p. 37) insiste en que una "nueva educación debe fracturar y resolver los errores mentales, intelectuales de la razón, las cegueras paradigmáticas y las convicciones y creencias que se han aprendido y que se llevan como lentes para percibir el mundo".

Al respecto, el examen crítico más general de Touraine (2000, p. 94) aboca el análisis del sujeto dando cuenta de cómo la preocupación cultural de promover procesos de subjetivación racionalista, que hizo originariamente de la modernidad un proyecto revolucionario, se transformó en racionalidad de control sobre los individuos, escindiéndolos internamente y dividiéndolos y oponiéndolos de cara al todo social, desconociendo otras dimensiones. De ese modo, "el agotamiento de la modernidad se transforma pronto en un sentimiento angustioso por la falta de sentido de una acción que ya no acepta como criterios, sino los de la racionalidad instrumental".

\section{Los quiebres en la construcción de un nuevo concepto de sujeto}

Conforme a lo dicho anteriormente, la posibilidad de construir un concepto de sujeto, que al mismo tiempo lleve a transformaciones sustanciales en lo educativo, y que impacte proyectos y políticas educativas que tengan un alto potencial constructor de relaciones de convivencia respetuosa, de equidad y calidad, pasa necesariamente por el quiebre de esta trilogía conservadora que, desde estas miradas, obstaculiza los cambios en los procesos educativos.

En atención a lo precedente, la primera ruptura ha de desplazar los criterios que definen al sujeto dentro de la cultura patriarcal neocapitalista a una cultura cuyo sujeto está sumergido en un mundo más solidario. El egoísmo espontáneo que todavía llevan los seres humanos, alimentado por el ansia de provecho y poder que segrega la mentalidad capitalista y consumista han conver- 
tido los debates sobre el sujeto en postulados individualistas. En el campo jurídico y político, el sujeto se utiliza como estandarte para la reivindicación de los propios derechos, como arma para exigir respeto individual. En el terreno psicológico el mismo concepto sirve para justificar el culto a la propia personalidad, con derecho a estructurarse caprichosamente y a expresarse en conductas que en ocasiones afecta a los demás degradándolos. En lo filosófico, el tema del sujeto no conduce muchas veces más que a acentuar los sentidos del individualismo, subjetivismo y fanatismo que lo mantienen abstraídos de los problemas que afectan la comunidad. De ahí la necesidad de reconocer que el sujeto es lo propio de todo hombre, no sólo mío. Al lado de un sujeto como yo, existe un tú (sujeto), un él (otro sujeto) y un nosotros (otros sujetos). Y cada uno de ellos es un sujeto; es decir, otro sujeto que merece respeto; que debe ser incluido, valorado y dignificado en la cultura donde vive. Se ha de tener en cuenta que ese sujeto, que esos sujetos, también son ciudadanos y están en posibilidad de construir lo público, de formarse para vivir democráticamente y aportar significativamente a procesos de transformación histórica. Que sus derechos fundamentales no deben ser vulnerados; que debe participar en iguales condiciones, de todos los bienes y servicios que el Estado debe brindarle. Es decir, una cultura solidaria e incluyente no trata el sujeto como un instrumento, sino como parte vital de un conjunto comunitario de sujetos capaces de autodeterminación. En palabras de Touraine, "resulta imposible separar al individuo de su situación social. Hay que oponer al individuo consumidor de normas y de instituciones sociales al individuo productor de esa vida social y sus cambios" (2000, p. 232).

Ahora bien, el segundo quiebre de lo que aquí se ha llamado, "el mito de la objetividad", surge en el ámbito de la pedagogía cuando, al lado de tales sujetos emerge una comunidad de sujetos diversos, pero al mismo tiempo llamados a construir aspectos comunes; unidad en medio de la diversidad. El sujeto como alte- 
ridad, abierto a los otros sujetos y constructor de nuevos mundos basados en acuerdos, en donde la autonomía florece como parte del tejido artesanal de un sujeto capaz de pensar por sí mismo en libertad, pero, al mismo tiempo, capaz de llevar al taller de las relaciones humanas, su propia verdad, su versión sobre el mundo, su conocimiento y subjetividad, que lo afecta a él y al entorno cultural donde vive.

Esta perspectiva es precisamente la que propone Habermas en una de sus principales tesis sobre la necesidad de la teoría de una acción comunicativa, si se quiere abordar hoy de una forma adecuada la problemática de la racionalización social, en el sentido de que la racionalidad no necesariamente tiene que ver con el conocimiento y menos con un conocimiento universal y absoluto, a los cuales los individuos tienen que aproximarse como seres pasivos.

Superando esa objetividad estricta se aborda la racionalidad desde la utilización comunicativa del saber proposicional en actos de habla tomando así una pre-decisión a favor de un concepto de racionalidad más amplio, que es el resultado de la capacidad de aunar conceptos sin coacciones y de generar el consenso que tiene un habla argumentativa en que diversos participantes superan la subjetividad inicial de sus respectivos puntos de vista, y a merced una comunidad de convicciones racionalmente motivadas que asegura a su vez la unidad del mundo objetivo y de la intersubjetividad del contexto en el que se desarrollan sus vidas. Así, de la manipulación instrumental se pasa al entendimiento comunicativo. Hay un desplazamiento de una visión "realista" que se limita a analizar las condiciones que un sujeto agente tiene que cumplir para poder proponerse fines y realizarlos en situaciones o "verdades" ya controladas, a una visión fenomenológica que no parte de un presupuesto ontológico de un mundo objetivo, sino que problematiza las situaciones preguntándose por las condiciones bajo las cuales se constituye para los miembros de una comunidad que lo 
reconocen por ser capaces de lenguaje y acción en un contexto que comparten. Habermas (2008), lo enuncia de la siguiente manera.

Las interacciones sociales son más o menos cooperativas o estables, más o menos conflictivas o inestables. La cuestión teórico-social de cómo es posible el orden social se corresponde con la cuestión de teoría de la acción de cómo al menos dos participantes en la interacción pueden coordinar sus planes de acción de forma que alter puede "enganchar" sus acciones en las de ego sin conflictos y, en todo caso, evitando el peligro de una ruptura de la interacción...El concepto de la acción comunicativa está pensado de tal manera que los actos del entendimiento que vinculan los planes de acción de diversos participantes y que resumen las acciones orientadas a un objetivo en una relación interactiva no pueden retrotraerse, por su parte, a una acción teleológica. Los procesos de entendimiento buscan un acuerdo que depende de la aprobación, racionalmente motivada, del contenido de una aseveración. No es posible imponer el acuerdo a la otra parte, ni se le puede imponer al interlocutor mediante una manipulación. El acuerdo descansa siempre sobre una convicción conjunta (pp. 138-139).

Abrirse al mundo del otro, para entender sus problemáticas y operativizar acciones conjuntas, es romper con un objetivismo que anula al otro. De ahí que, el sujeto, si realmente tiene algún valor para el mundo de hoy, exige prestarle atención. El sujeto como ser personal se manifiesta a través de la palabra. Ésta, en su significado profundo y existencial, revela al otro como referente, importante y activo en la construcción del devenir histórico, por eso hay que estar pendiente de su palabra, de lo que dice, de lo que piensa, de lo que hace, para crear puentes de diálogo entre sujetos libres, no ya desde el punto de vista comercial o mercantil, sino desde un encuentro de apertura y crecimiento. Hay que estar atentos, por tanto a los otros sujetos, a sus palabras, que aunque no son iguales a las de otros sujetos, y les pueden resultan extrañas, a 
veces irreverentes y revolucionarias, con relación a las palabras de los demás, son importantes. En términos de Ibáñez (1998, p. 56), la educación ha de comprender que "el sujeto está sumergido en el orden simbólico, orden del metabolismo social; del intercambio de objetos, se sujetos y de mensajes", que constantemente lo están enriqueciendo.

Hoy es necesario considerar lo subjetivo y lo objetivo como parte crucial de la concepción del concepto de sujeto; lo cual es fundamental para tejer los procesos educativos que se puedan implementar, en la medida en que permite la construcción de la "objetividad" a través de los consensos entre sujetos libres y autónomos; y las reflexiones continuas que los ciudadanos hacen sobre un asunto para elegir cuál es la salida más conveniente para todos, de cara a los diferentes conflictos socio-educativos que viven las sociedades. Y es que, como lo señala Arendt (2006, p. 207) "la esfera de los asuntos humanos, estrictamente hablando está formada por la trama de las relaciones humanas que existe donde quiera que los hombres viven juntos y son capaces de ponerse de acuerdo". Ese interés común se da gracias a una de las dimensiones generativas del lenguaje: la reflexión, entendida como el análisis individual de los argumentos propios y ajenos de los sujetos involucrados, para darse cuenta, para entender y dar razón de lo que se quiere y de lo que no se quiere. Se trata de una actitud libre y espontánea que permite superar los conceptos unívocos del mundo, en una actitud crítica y con sentido de transformación y planeación de nuevas relaciones en donde lo pedagógico cumple un papel muy importante.

La tercera y última ruptura está en relación con el desarrollo del sujeto emocional. Betancourt y Pavajeau (2007) piensan que:

Ante el sesgo que ha promovido para la educación, el paradigma racionalista- mecanicista y ante el mundo desconcertante y desafiante que hoy y cada vez presenciamos, reconocer y 
educar también la inteligencia emocional, parece ser pues, una buena alternativa, primero, para una mejor aproximación a una verdadera educación integral y segundo, para contribuir de una manera más legitima, a construir un mundo y una sociedad que tenga como propósito central y esencial del desarrollo, en función de la mayor dignificación, respecto y felicidad del ser humano (p. 16).

La insistencia en identificar la razón como la facultad que manifiesta la superioridad del hombre sobre todas las demás especies, ha hecho olvidar que los seres humanos también son seres emocionales (afectivos). Son muchos los factores que han contribuido a fortalecer el énfasis en lo racional. La cultura occidental ha sido siempre dominada por el varón, quien en ciertos ambientes se jacta de su inteligencia calculadora y deja para la mujer lo afectivo. El racionalismo del mundo moderno impulsado por Descartes, encontró el terreno abonado para sembrar el culto a la razón que florecerá en la época de la ilustración. Es así como se ha llegado a construir una cultura racionalista y fría en la que los sujetos, sin darse cuenta, bailan vertiginosamente al ritmo de las máquinas y los mecanismos de producción y consumo, donde lo único que cuenta es "saber". Quien sabe es útil, quien ama es inútil. Lo afectivo ha quedado arrinconado a la vida del hogar o a ciertos momentos puntuales. Por eso, bien lo ha sostenido Goleman (1996, p. 22) al pensar que "una visión de la naturaleza humana que pasa por alto el poder de las emociones es lamentablemente miope". Es urgente devolver al sujeto actual, que ha progresado en el terreno del conocimiento, el sentido profundo del amor en todas sus manifestaciones, amistad, erotismo, caridad, etc.

El que la afectividad y el amor no se hayan desarrollado en la humanidad, al mismo ritmo que los conocimientos, constituye un grave peligro que amenaza la identidad del sujeto social. Vivir más preocupados por las cosas que por los demás sujetos ha creado sociedades violentas y guerristas. Al parecer, al sujeto de hoy le 
conmueve más la pérdida de una fórmula científica, de un libro, de unas elecciones parlamentarias, que el asesinato de una persona, la masacre de un pueblo o el hambre de muchos seres humanos.

En el campo educativo lo afectivo-emocional, cumple un papel fundamental y decisivo para la formación del sujeto, como lo advierte De Zubiría (2006, p.88), en la medida en que "aporta las motivaciones, las actitudes, los intereses y las expectativas que echan a andar, orientan, dirigen y sostienen al sistema cognitivo". Y, aún más, Antunes (2005, p.25) ha considerado que "la evolución de nuestra especie dio a la emotividad un papel esencial para la solución de los problemas más prominentes de la vida. Es ella la que nos conduce cuando surgen desafíos esenciales que no pueden ser resueltos por nuestra pobre intelectualidad".

En este sentido, el desarrollo de la vida emocional en la formación del sujeto es sustancial en la medida en que crea los escenarios adecuados y pertinentes para provocar cambios que no dependen de estructuras que exigen un deber como cumplimiento de una serie de normas que el sujeto debe seguir, sino como procesos de gratificación emocional, que resultan de un cambio auténtico que parte del compromiso individual, el cual se da cuando la motivación e intereses son colectivos y comunes a los sujetos. Y en especial, cuando la gratificación se centra en la acción misma y no en sus resultados. En este caso lo que se disfruta es la nueva manera de vivir la cotidianidad, lo cual tiene un afecto transformador al reforzar y consolidar los cambios, imprimiéndoles permanencia cronológica.

Es cierto que en ocasiones el sujeto cumple con un deber que no le satisface, pero que le toca llevarlo a cabo, por temor a ser "castigado"; en cambio, esta nueva perspectiva contempla aquellos cambios que se producen al interior de la conciencia del sujeto y que le generan gratificación, en la medida en que racionalmente los han entronizado, reflexionado y comprendido, y emocionalmente siente gusto en realizarlos. 


\section{Conclusiones}

Numerosas pruebas anatómicas y fisiológicas ${ }^{1}$ han venido demostrando que pensar y sentir, es decir, el cerebro racional y emocional, forman una unidad inseparable. Para poder comprender e interpretar el entorno en que se desenvuelven los sujetos se necesitan ambas cosas: la inteligencia racional y planificadora y el mundo de las emociones que suele actuar de un modo más bien espontáneo. Sólo la coordinación de la capacidad de sentir y la capacidad de pensar otorgan al sujeto su amplio abanico de posibilidades en la búsqueda de cambios significativos que permitan bajar del pedestal el mito de la razón y abran también el campo emocional como constitutivo de su esencia, de manera que, habida cuenta de esa relación en los procesos de interacción del sujeto con otros sujetos, se destruya, de la misma manera, el mito objetivista y se abran así las puertas para la construcción de sociedades más pluralistas en donde el sujeto particular entre a participar con criterio en el tejido de los consensos. Desde esa perspectiva, la construcción de la convivencia democrática sólo será posible mediante un cambio

1 Desde los años 30 del siglo XX se impulsó toda una serie de experimentos, especialmente en los Estados Unidos y en Europa, que involucraron trabajos con pacientes individuales, con grupos de escolares, con personas que se ofrecieron, a través de diversas encuestas que se hicieron, etc. Todo ello para estudiar el comportamiento de cerebro humano y conocer cómo opera en diversas situaciones y cuál es el papel del cerebro emocional y del cerebro racional y la forma en que se integran. Una de las grandes conclusiones, entre otras tantas, es que, definitivamente el cerebro emocional actúa primero que el racional y éste da cuenta del sentido y significado de lo que los seres humanos sienten o experimentan. Se puede citar, en otros, los trabajos de Goleman (1995), Damasio (1996), Maturana (1993), Asensio (1997), Feixas (1993), Gergen (1996), Greenberg y Paivio (1999), Davinson (2002), Baving (2002), LeDeux (1994), Salovey (1990), Chabot (1996), Labarbera (1976), Field (1982), Gosselin (1995), Berry (1993), Lane (1998), Bischof (1994), Reynolds (1999), Hughes (2000), Nowicki (1989), Azor (1981), Coffman (1981), Ekman (1983), Strack (1988), Laird (1974), Blosh (1989), Benson (1975), Jevening (1978), Delmonte (1985), McCraty (2002), Morgan (1976), Colt (1981), Stepton (1996), Ver Lazarus (1991), Tomarken (1990), Aftanas (2001), Malathi (1999), Shah (2001), Shapiro (1998), Cormier (1996), Gottman (2000), Boyatzis (2002), Breur (2003), Choi (2003), Assor (2002). 
en el emocionar de los sujetos que desean realizarla, el cual se debe extender a todos los dominios de la relaciones humanas, creando espacios en los que el acuerdo, la cooperación y la reflexión reemplacen a la autoridad, el control y la obediencia como maneras de coexistencia humana, de modo que se puedan tejer redes de conversaciones entre sujetos que se sienten en iguales condiciones. Así, sólo es posible acabar también con el mito de la cultura patriarcal, que ha hecho tanto daño a la humanidad, y abrir caminos de esperanza en la lucha por conquistar sociedades más equitativas, más justas y menos violentas. Será, pues la tarea del sujeto, de los Estados, de los grupos y de las culturas en general para estos albores del siglo XXI.

\section{Bibliografía}

ARENDT, Hanah. (1998). La Condición Humana. Barcelona, Ediciones Paidos.

BALBÍN, Jesús William y ARANGO, Oscar Ignacio (2003). Globalización de los Derechos. Repensando la Condición Humana. Medellín, IPC de la corporación de promoción popular.

CELSO, Antunes. (2005). Educar en las Emociones. Nuevas Estrategias para el Desarrollo de las Inteligencias Múltiples. Buenos Aires, Ediciones San Benito.

DE ZUBIRÍA S., Miguel (2006). El Mito de la Inteligencia y los Peligros del Cociente Intelectual. FIPC. Bogotá, Instituto Internacional Alberto Merani.

ECHEVERRÍA, Rafael (1996). Ontología del Lenguaje. Santiago, Dolmen.

GOLEMAN, Daniel (1996). La inteligencia emocional. Buenos Aires, Javier Vergara Editor.

HABERMAS, Jürgen (2008). Conciencia Moral y Acción Comunicativa. Madrid, Editorial Trotta. 
IBÁÑEZ, Jesús (1998). Nuevos Avances en la Investigación Social I. Barcelona, Ediciones Proyecto A.

MATURANA, Humberto (2002). La objetividad. Un argumento para obligar. Santiago, Océano-Dolmen.

MATURANA, Humberto y De Rezepka, Nisis (2003). Amory juego. Fundamentos olvidados de lo humano. Santiago, UNICEFDolmen.

MÄRTIN, Doris y Boeck, Karin (2007). Qué es inteligencia emocional. Madrid, Improve.

MAYA BETANCOURT, Arnobio y PAVAJEAU D., Nohora Cecilia (2007). Inteligencia emocional y educativa. Una necesidad humana curricular y práctica. Bogotá, Editorial Magisterio.

MORIN, Edgar (1996). El pensamiento complejo. Barcelona, Gedisa.

PERESSON, Mario (2005). Análisis de estructura y coyuntura. Bogotá, Colección experiencias.

TOURAINE, Alain (2000). Crítica de la Modernidad. Bogotá, Fondo de Cultura Económica.

Recibido en noviembre 2010 ReEnviado enero ZOII Arbitrado febrero 2OII 